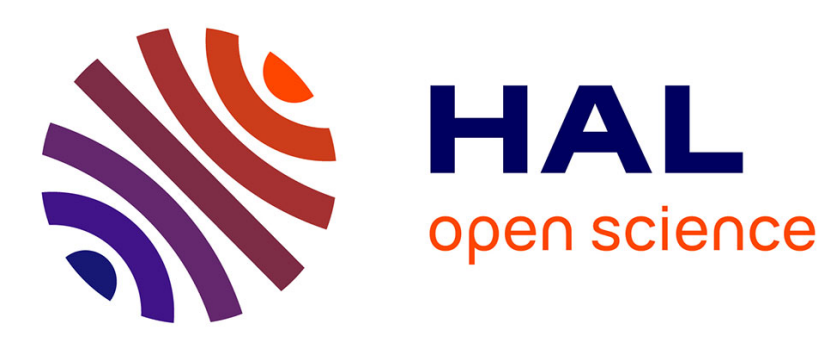

\title{
Probing machine-learning classifiers using noise, bubbles, and reverse correlation
}

Etienne Thoret, Thomas Andrillon, Damien Léger, Daniel Pressnitzer

\section{To cite this version:}

Etienne Thoret, Thomas Andrillon, Damien Léger, Daniel Pressnitzer. Probing machine-learning classifiers using noise, bubbles, and reverse correlation. Journal of Neuroscience Methods, 2021, 362 (109297), 10.1016/j.jneumeth.2021.109297 . hal-03063763

\section{HAL Id: hal-03063763 https://hal.science/hal-03063763}

Submitted on 7 Jan 2021

HAL is a multi-disciplinary open access archive for the deposit and dissemination of scientific research documents, whether they are published or not. The documents may come from teaching and research institutions in France or abroad, or from public or private research centers.
L'archive ouverte pluridisciplinaire HAL, est destinée au dépôt et à la diffusion de documents scientifiques de niveau recherche, publiés ou non, émanant des établissements d'enseignement et de recherche français ou étrangers, des laboratoires publics ou privés. 


\section{Probing machine-learning classifiers using noise, bubbles, and}

\section{2 reverse correlation}

3

4 Etienne Thoret ${ }^{* 1,4}$, Thomas Andrillon ${ }^{3}$, Damien Léger ${ }^{2}$, Daniel Pressnitzer $^{1}$

5

$6 \quad{ }^{1}$ Laboratoire des systèmes perceptifs, Département d'études cognitives, École normale supérieure, PSL University, CNRS, 75005 Paris, France.

${ }^{2}$ Université de Paris, APHP, Hotel Dieu, Centre du Sommeil et de la Vigilance \& EA 7330 VIFASOM, Paris 75006, France.

${ }^{3}$ Turner Institute for Brain \& Mental Health and School of Psychological Sciences, Monash University, Melbourne 3168, Australia.

${ }^{4}$ Aix Marseille Univ, CNRS, PRISM, LIS, ILCB, Marseille, France *corresponding author: etiennethoret@gmail.com 


\section{Abstract}

Many scientific fields now use machine-learning tools to assist with complex classification tasks. In neuroscience, automatic classifiers may be useful to diagnose medical images, monitor electrophysiological signals, or decode perceptual and cognitive states from neural signals. Tools such as deep neural networks regularly outperform humans with such large and high-dimensional datasets. However, such tools often remain black-boxes: they lack interpretability. A lack of interpretability has obvious ethical implications for clinical applications, but it also limits the usefulness of machine-learning tools to formulate new theoretical hypotheses. Here, we propose a simple and versatile method to help characterize and understand the information used by a classifier to perform its task. The method is inspired by the reverse correlation framework familiar to neuroscientists. Specifically, noisy versions of training samples or, when the training set is unavailable, custom-generated noisy samples are fed to the classifier. Variants of the method using uniform noise and noise focused on subspaces of the input representations, so-called "bubbles", are presented. Reverse correlation techniques are then adapted to extract both the discriminative information used by the classifier and the canonical information for each class. We provide illustrations of the method for the classification of written numbers by a convolutional deep neural network and for the classification of speech versus music by a support vector machine. The method itself is generic and can be applied to any kind of classifier and any kind of input data. Compared to other, more specialized approaches, we argue that the noise-probing method could provide a generic and intuitive interface between machine-learning tools and neuroscientists.

Keywords: Data analysis - Interpretability - Deep neural networks - Automatic classifiers - Reverse correlation - Auditory models 


\section{Introduction}

Applications of machine-learning techniques permeate more and more scientific fields, with rapid and sometimes unexpected success (LeCun et al., 2015; Jordan \& Mitschell, 2015; Krigeskorte \& Douglas, 2018; Richards et al., 2019). At the same time, it is becoming a widely-acknowledged issue that many of these tools are often used as black boxes, and need to be interpreted (Molnar, 2020; Doshi-Velez \& Kim, 2017). For instance, if a Deep Neural Network (DNN) was used to make lifechanging decisions such as deciding on an intervention based on medical imagery, both the clinicians and patients would have a clear desire to know the rationale that motivated the decision. Also, the power of classifiers to detect useful information in large datasets holds many promises to improve theoretical models, but then, understanding at least to some extent the classifier's operation is crucial (Zihni et al., 2020).

Understanding what a complex classifier does after being trained on possibly millions or billions of samples is usually hard. It is hard for a reason: if the task that the classifier solves had a known explicit solution, then there probably would not have been any incentive to develop the classifier in the first place. In addition, modern techniques involve artificial network architectures with interconnected layers, each including highly non-linear operations (Sejnowski, Kienker, \& Hinton, 1986). A lot of the computational power of such algorithms lies in such cascades of feedforward and feed-back non-linear operations. Unfortunately, human reasoning seems most at ease to generate intuitions with linear processes, and not for complex combinations of non-linear ones.

As a consequence, designing methods to interpret machine learning tools is a fast-growing field of research of its own right, often designated under the term Explainable AI (Guidotti et al., 2018). It has dedicated journals within the machine learning community (e.g. Distill) and an associated DARPA challenge (XAI). Recent reviews covering the types of methods exist (Molnar, 2020), also covering more specifically the feature visualization approach taken here (Olah et al., 2017). Within this context, our aim is not to outperform the state-of-the art specialized interpretability methods, but rather to provide a general tool that will hopefully be intuitive to neuroscientists, as it is based on familiar methods for this community. The manuscript describes the method, provides an open 
bioRxiv preprint doi: https://doi.org/10.1101/2020.06.22.165688; this version posted June 23, 2020. The copyright holder for this preprint

(which was not certified by peer review) is the author/funder, who has granted bioRxiv a license to display the preprint in perpetuity. It is made available under aCC-BY-ND 4.0 International license.

software library to use it, and shows examples of application, demonstrating how it can achieve useful results.

The gist of the method is to try and reveal the input features used by an automatic classifier, a black-box, to achieve its task without any knowledge about what is inside the black-box. As such, it is what is termed an "agnostic" method of explanation: it does not attempt to describe mechanistically the operation of a specific classifier, which it considers unknown (even if the classifier's details are available, as they may be too complex to understand intuitively). Rather, the aim is to relate features of the input space to the classifier's decisions. Such a problem is closely related to issues that neuroscientists and experimental psychologists have been addressing for years: providing useful insights for theoretical models of, for instance, human perception, without a full knowledge of the highly complex and non-linear underlying information processing performed by the brain. In particular, the method we propose is directly inspired from the reverse correlation techniques developed for studying human vision (Ahumada et al., 1971; Neri et al., 1999; Gosselin \& Shyns, 2001, 2003). Reverse correlation is based on linear systems analysis (Wiener, 1966). It uses stochastic perturbations of a system to observe its output. If the system were linear, an average of the inputs weighed by the observed outputs would be able to fully characterize the system. However, even for the highly non-linear systems studied by neuroscience, reverse correlation has a track-record of useful applications. For neurophysiology, averaging input stimuli according to neural firing rates has been used to describe neural selectivity (Ringach \& Shapeley, 2004 for a review). For psychophysics, averaging input stimuli according to participant's decisions has revealed stimulus features on which such decisions are made for detection or discrimination tasks (Ahumada et al., 1971; Gosselin \& Shyns, 2001, 2002). In this spirit, it seems appropriate to add reverse correlation to the toolbox of techniques to probe automatic classifiers, as its advantages and limitations are already well understood for non-linear systems.

One important benefit of using the reverse correlation framework is its complete independence from the underlying classifier's architecture. Unlike efficient but specific methods tuned to a classifier's architecture (see Guidotti et al., 2018 for a review), the reverse correlation can be used to probe any algorithm that separates the input data into distinct classes. Even for the 
93 currently popular agnostic interpretability methods, this is not always the case: Class Activation

94 Maps (Zhou et al., 2016) are specific to convolutional networks; LIME (Ribeiro et al., 2016) and

95 RISE (Petsiuk et al., 2018) highlight features of specific examples which may or may not be

96 representative of the classification task in general. Also, the method operates in the same

representation space used as an input to the classifier, and can be applied to any type of instance).

The outline of the method is as follows. First, a set of stochastic inputs are generated, by introducing noise on the training dataset when available, or, when unavailable, by generating broadband noise to cover systematically the input space. The noise takes two forms: additive noise, as is classically the case, but also multiplicative low-pass noise known as "bubbles" (Gosselin \& Shyns, 2001, 2002) to focus the exploration on sub-spaces of the input representation. Second, the inputs are sorted according to the classification results. Third, inputs belonging to the same class are grouped together, with some refinements of the standard reverse correlation methods inspired by signal necessarily identical input features: (1) the discriminative features, which correspond to the part of the input representation that is the most useful to ascribe a category (2) the canonical features, which literature, these would loosely correspond to the "attribution" versus "feature visualization" problems (Ohla et al., 2017). In psychophysics, the distinction overlaps with the "potent information" (Gosselin et al., 2001) versus "prototypical information" (Rosch, 1983).

\section{Material and Methods}

\subsection{Probing discriminative features}

We term "discriminative features" the subspaces of the input space that are the most potent in 
bioRxiv preprint doi: https://doi.org/10.1101/2020.06.22.165688; this version posted June 23, 2020. The copyright holder for this preprint (which was not certified by peer review) is the author/funder, who has granted bioRxiv a license to display the preprint in perpetuity. It is made available under aCC-BY-ND 4.0 International license.

120 visualize such subspaces in the input space. In the following, we assume that the classifier has been

121 trained and is available to the probing method.

122

123

124

125

126

127

\subsubsection{Procedure}

To identify discriminative features, the input space is pseudo-randomly sampled with multiplicative low-pass filtered noise. The subspace enabling the highest classification performance is then identified by a reverse correlation analysis of all classified samples. The algorithm is directly inspired by the "bubbles" method (Gosselin \& Shyns, 2001), originally designed to characterize the visual features underlying human behavioral performance for image classification tasks.

We present two sub-variants of the method, to account for the availability or not of the training set: a) multiplicative lowpass noise is applied to the training set; 1b) multiplicative lowpass noise is applied to broadband noise generated in the input stimulus space. We now describe the algorithm, jointly for a) and b). A textual description is provided as well as a software repository written in Python programming language (https://github.com/EtienneTho/proise) and a schematic illustration (Figure 1).

\section{Method 1: Discriminative features}

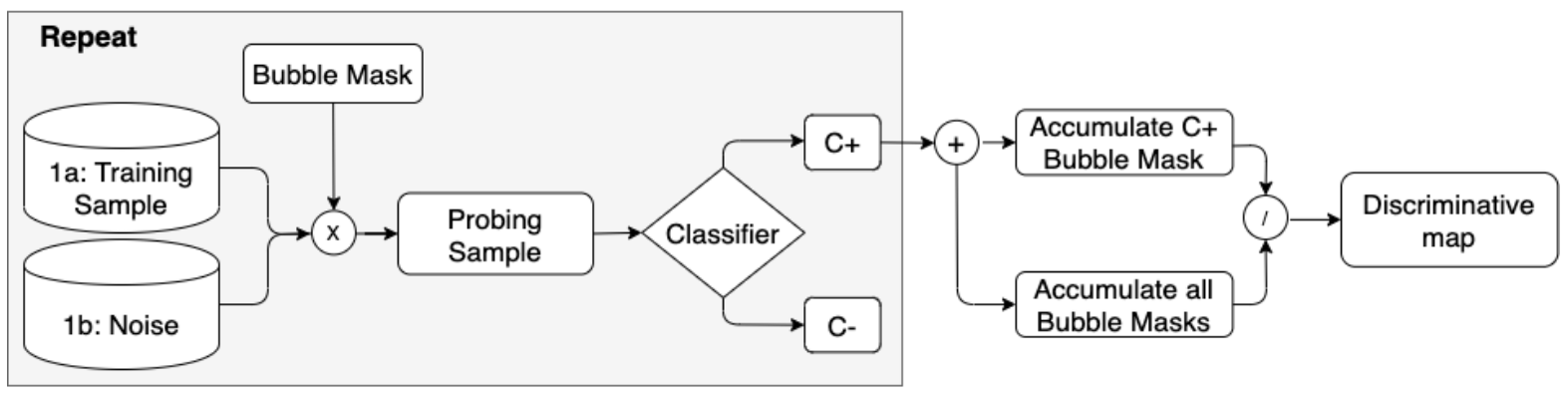

\section{Method 2: Canonical features}

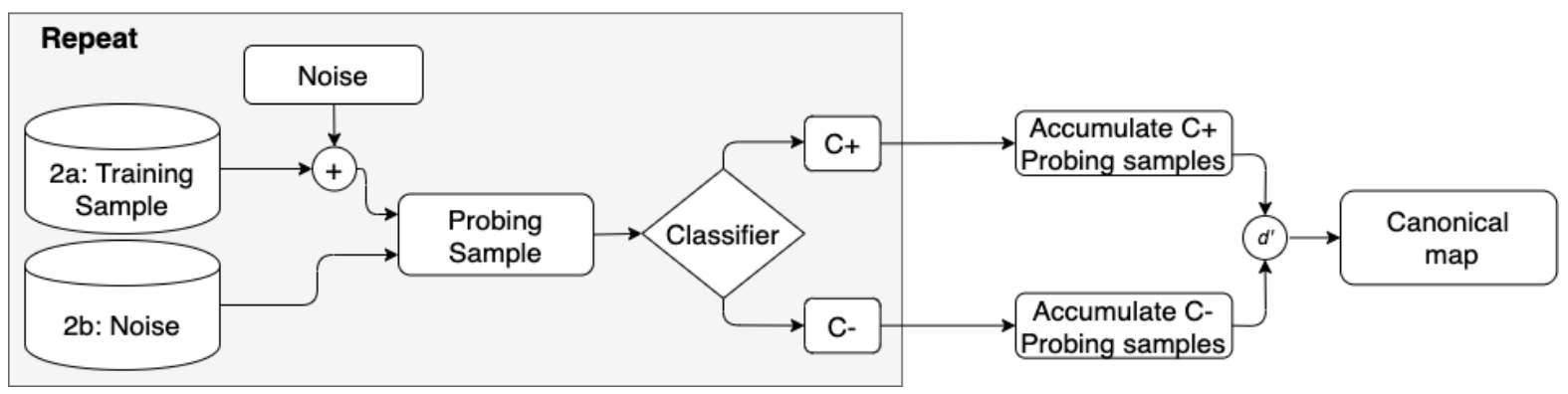

Fig. 1. Summary of the two probing methods. Both method have 2 variants depending on the availability of the training set. Method 1: Training samples (1a) or noise (1b) are multiplied by bubble masks and then fed to the trained classifier. The bubble masks are then sorted according to 
bioRxiv preprint doi: https://doi.org/10.1101/2020.06.22.165688; this version posted June 23, 2020. The copyright holder for this preprint (which was not certified by peer review) is the author/funder, who has granted bioRxiv a license to display the preprint in perpetuity. It is made available under aCC-BY-ND 4.0 International license.

the output of the classifier to compute the discriminative map. Method 2: Training samples with additive noise (2a) or noise (2b) are fed to the trained classifier. The probing samples are then sorted according to the output of the classifier to compute the canonical map. For methods $1 b$ and $2 b$, the noise can be either a gaussian noise or pseudo-random samples.

For each pass (gray box in Fig. 1):

1. A bubble mask is generated. This consists of a mask in the input space, of dimension $\mathrm{N}$, consisting of randomly positioned N-dimensional Gaussian windows (see Figure 2). The number of bubbles, nbBubbles, as well as the size of the bubbles in terms of the Gaussian standard deviations can be arbitrarily chosen and are parameters of the algorithm. In practice, an input array of dimension $\mathrm{N}$ populated by zeroes except for $n b B u b b l e s$ unit values is convolved with $\mathrm{N}$-dimensional Gaussian windows. The resulting mask is denoted BubbleMask.

2. The probing data is generated. For variant a), the probing data is one exemplar of the training dataset, randomly chosen. For variant b), the probing data is an N-dimensional activation noise (see section 2.1.2 for details). The probing data is denoted ProbingData.

3. The probing sample is obtained by multiplying the bubble mask with the probing noise: ProbingSample $=$ BubbleMask $*$ ProbingData.

4. The probing sample is fed to the classifier and the output class is recorded. The probing sample is labeled $\mathrm{C}+$ if it classified in the target class, $\mathrm{C}$ - otherwise.

Analysis:

For each point, $i$, in the stimulus space, the discriminative map for the class $\mathrm{C}+, D_{\mathrm{i}, \mathrm{C}+}$, is computed as the sum of all $\mathrm{C}+$ bubble masks divided by the sum of all masks $\mathrm{C}+$ and $\mathrm{C}-\mathrm{:}$ $D_{i, C+}=\frac{\sum \text { BubbleMask }_{C+, i}}{\sum \text { BubbleMask }_{i}}($ Eq. 1). It should be noted that the analysis is performed on the bubble masks, and not on the probing samples.

\subsubsection{Generation of noise activations when the training set is unavailable}


As mentioned above, when the training set of the classifier is unavailable, the probing

samples are generated from noise in the input space. The choice of the noise distributions is a free

parameter of the method. The simplest choice is to draw samples from a uniform distribution at each

point of the input space, covering the full range of valid input values. However, this sometimes leads

to uneven coverage of the output categories, for instance if the classifier's boundaries are especially

Analysis (PCA). As the training set is unavailable, the PCA can be done on a representative set of inputs relative to the classifier's task. After the PCA, uniform noise can be generated in the low dimension space - which can be seen as the latent PCA's space - and inverted to obtain noise in the input space. The qualitative goal during the choice of the noise distribution is a balanced coverage of all output categories, and iterative choices may be a part of understanding the classifier's features.

\subsubsection{Statistics} of each part of the discriminative map.

There are many options to assess significance of such data, from which we outline one above many times while randomly assigning output categories to each sample. For each point in the actual map, a $t$-test (or a non-parametric equivalent) is applied to compare the map value with the mean of the shuffled data. Maps are usually high-dimensional so a correction for multiple comparisons is needed. Again, several choices exist which are not specific to the methodology 
We define "canonical features" as the representation, in the input space, that would best match

the different items of a given class. As an analogy, the canonical information may be viewed as the centroid of a category in the input space.

\subsubsection{Procedure}

To build the canonical map, the whole input space is randomly perturbed, without bubbles, so

the search is not focused on any subspace. The aim is to probe the whole feature space. Then, all

probes classified as members of the same category are averaged, in a direct adaptation of the classic

reverse correlation method. However, we introduce here two differences, though. First, for

generality, we do not separate correct classifications from false positives or false negatives. This

would require to know the training dataset or to have a large labeled testing dataset. Second, a

normalization of the feature map is introduced, using standard deviations estimates at each point of

Again, we propose two sub-variants of the algorithm depending on the availability or not of the

training dataset: a) broadband noise in the input space is added to the training set; b) broadband noise

is generated in the input space. We now describe the algorithm, jointly for a) and b). A textual

description is provided as well as the scripts (https://github.com/EtienneTho/proise) and a schematic description (Figure 1).

For each pass:

1. The probing sample is generated. For a), the probing sample is one randomly chosen exemplar of the training dataset, with noise added. The goal is to perturb the input to introduce variability, so that only the most salient information (to the classifier) remains in the reverse correlation average. For b), the probing sample is an $\mathrm{N}$-dimensional activation noise. The probing sample is denoted ProbingSample.

2. The probing sample is fed to the classifier and the output class is recorded. The probing sample is labeled $\mathrm{C}+$ if it classified in the target class, $\mathrm{C}$ - otherwise.

Reverse correlation analysis: 
- For each point, $i$, in the stimulus space, the discriminative information is computed as the mean of all $\mathrm{C}+$ probing samples minus the mean of all $\mathrm{C}$ - probing samples, normalized by the standard deviation of all probing samples at this point in the input space:

$$
P_{i}=\frac{\text { mean }\left(\text { ProcingSample }_{i, C+}\right)-\text { mean }\left(\text { ProbingSample }_{i, C-}\right)}{\text { std(ProbingSample })} \quad \text { (Eq. 2) }
$$

This reverse correlation definition adds a normalization factor to the simple average, using the standard deviation observed over all probing samples. This normalization is inspired from the discriminability index $d^{\prime}$ of signal detection theory (Green \& Swets, 1966). It aims to visually emphasize reliably high values in the canonical map, by transforming the input units to $z$-score units.

Note also that in a binary classification task, $P_{\mathrm{i}}$ is symmetric for the two classes.

\subsubsection{Dimensionality reduction}

Depending on the architecture of the classifying pipeline and input space, the estimation of the canonical map with reverse correlation can be more or less efficient. In particular, a standard technique to improve efficiency when training a classifier is to reduce the number of dimensions of the input space, for instance by using PCA. (e.g., Patil et al., 2012). Here as well, the probing and reverse correlation analysis can be performed in the space with reduced dimensionality before inverting back to the original input space.

For the generation of probing noise in variant b), the same remarks made in section 2.1.2 apply, with the same use of PCA to shape the noise for a balanced coverage of all output classes.

\subsubsection{Statistics}

The statistical analysis of canonical maps can be done with the same tools as for discriminative maps, described in section 2.1.3.

\section{Results}

To illustrate the methods introduced above and their generality, we present two different use cases: interpreting the classification of handwritten digits, a visual task (2-D input space) performed with a deep neural network; interpreting the classification of speech versus music, an audio task (1-D time series converted to a 4-D auditory model) performed by a support vector machine. These two cases also cover binary versus multiclass decisions. Although voluntarily simple, these examples should cover most of the ingredients needed for use cases relevant to neuroscience, such as vocal 
246 classification (Paquette et al., 2018), biomedical images classification (Wang et al., 2016), EEG

247 decoding (King \& Dehaene, 2015), Multi-Voxel Pattern Analysis (Formisano et al., 2008).

\subsection{Digits classification}

249 In this first example, we classify visual samples of handwritten digits from the MNIST database

250 (Deng, 2012). This is a standard database for evaluating image classification algorithms in the

251 machine-learning community. It is composed of handwritten digits, from 0 to 9, with 60000 samples

252 in the training set and 10000 samples in the test set. Each sample is a two-dimensional greyscale

253 image with pixels values between 0 and 1.

254 Many algorithms can now successfully perform this classification task. Here we trained a

255 Convolutional Neural Network (CNN) to discriminate between digits, with the following

256 architecture: 2D-convolutional layer (3, 3), Max Polling layer (2, 2), 2D-convolution layer (3, 3),

257 flattening layer, dense layer with 10 outputs and a softmax activation. Three epochs were run and, as expected, a high classification accuracy of $97 \%$ was obtained on the test set. 


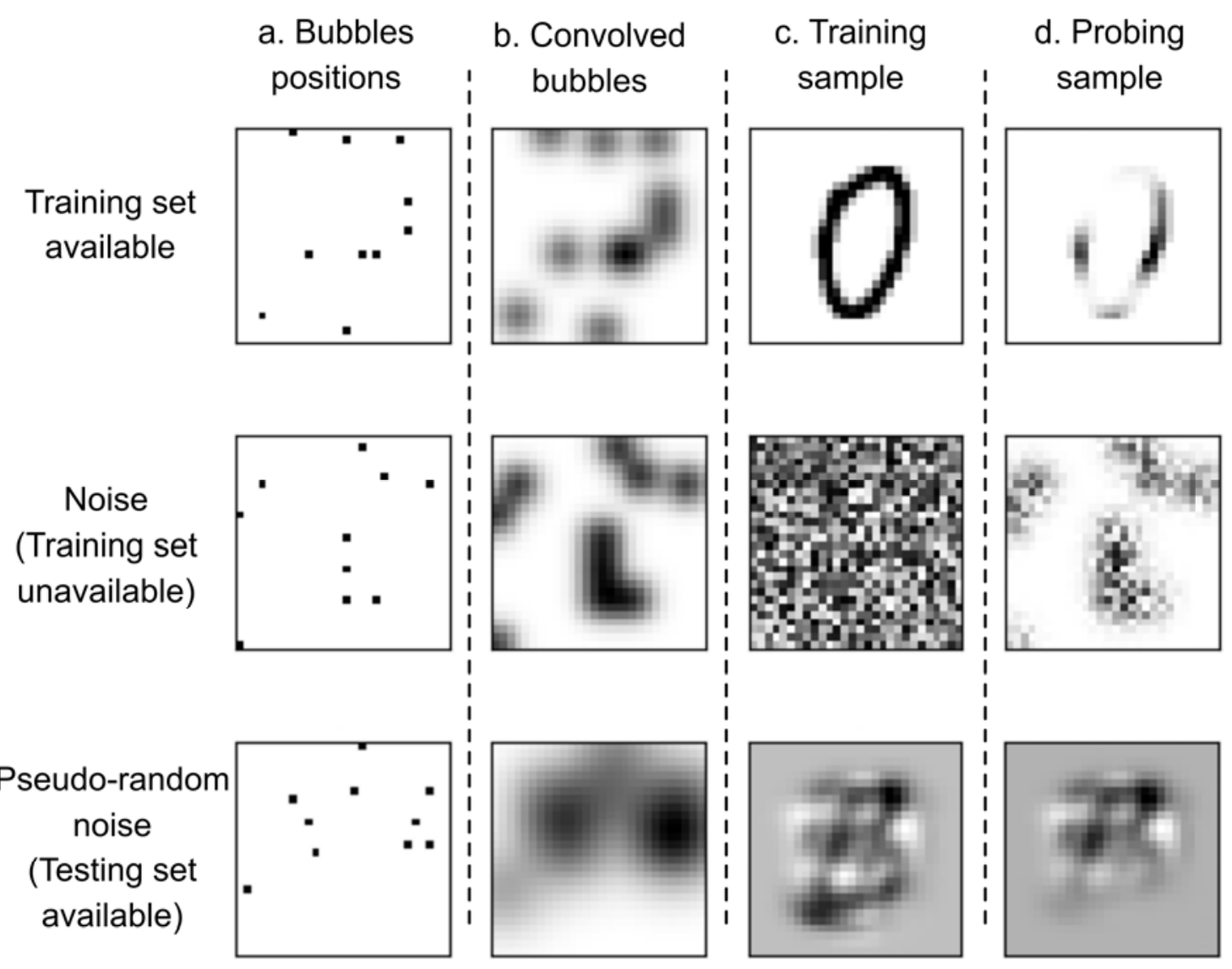

Fig. 2. Illustration of the probing method with bubbles on 2-D images. Top row: construction of

row: discriminative features with training set unavailable, uniform noise. Columns as above.

Using such probing samples do not cover the output categories efficiently (see text), likely because of shares visual features with actual digits.

Figure 3 shows the discriminative features obtained with Methods 1a) and 1b), expressed as the Discriminative Index of Eq. 1 visualized in the 2-D input space (the visual image). The resulting 
277 classification, that is, the position of the bubbles most useful for identifying each class. These do not

278 need to correspond to the actual shape of a digit (which will be targeted by canonical features later

279 on). For example, for the digit "1", the most useful regions are around the digit: knowing that there

280 are no active pixels in such surrounding regions is most efficient for deciding that the narrow-shape

281 of "1" was the input. For the digit "7", the discriminative map highlights the top-right corner, which

282 corresponds to the position of a sharp angle unique to "7". In summary, while these maps may not

283 make immediate intuitive sense on their own, they do orient the analysis of the input set towards

284 regions of interest. Moreover, if the task was now to classify "7" versus all other digits, the input

285 space could be weighed to emphasize the top-right corner to simplify the new classifier.

The availability of training data is expected to provide faster and more robust convergence

towards the features of interest. For each case, 60000 probing samples and 10 bubbles with standard

but only lead to categorization in 5 digits categories, so a pseudo random noise obtained from the

covering the 10 categories. The discriminative information obtained in the two cases correlate 

available under aCC-BY-ND 4.0 International license.

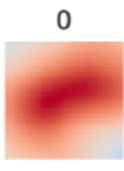

3

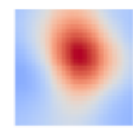

6

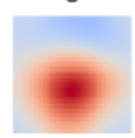

9

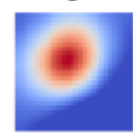

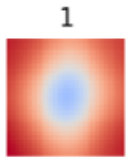

4

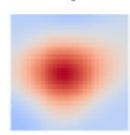

7

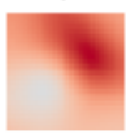

2

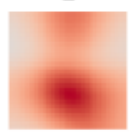

5

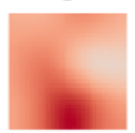

8

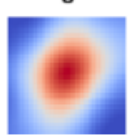

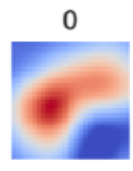

3

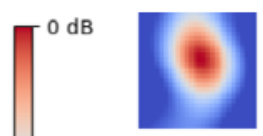

6

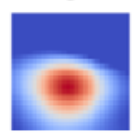

9

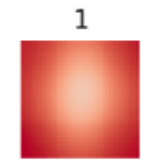

4

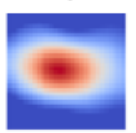

7

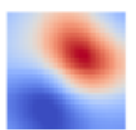

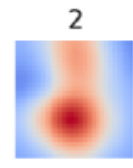

5

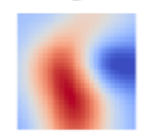

8

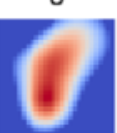

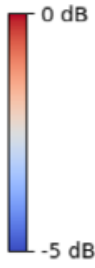

298

299

300

Fig. 3. Discriminative features maps for a CNN classifying handwritten digits. The maps show the

301

302

303

304

305

306

307

308

309

310

311

312

313

314

315

316

317

discriminative maps, in $\mathrm{dB}$, obtained for each digit with Method 1a) with the training set available (left) and Method 1b) with the training set unavailable (right). Regions in red correspond to subspaces of the input most important for a correct classification of each digit. The maps are here normalized for each digit and presented in $d B\left(20 \log _{10} P_{i} / P_{\max }\right)$ for a sake of comparability between digits.

Figure 4 shows the canonical features obtained with Methods 2a) and 2b), with or without the training set available. These canonical maps look different from the discriminative ones. Here, the maps are weighted averages of probing samples themselves, and not low-pass bubble masks, so finer details are available. As a result, and as intended with a reverse correlation approach, the canonical maps are readily identifiable and visually resemble the average written digits' representation. Such insight is perhaps not very surprising with simple digits, except perhaps for the 'negative' regions in

blue that further specify which features are canonically absent from a given digit. Again, Methods 2a) and $2 \mathrm{~b})$ provide strongly correlated maps $\left(r=.67(\mathrm{SD}=.18), d f=783, p<10^{-3}\right)$. It can nevertheless be noted that Method 2b) tends to focus on the center of the input space. In particular, some border pixels were never associated with one or the other classification decision, leading to missing values when computing $d$ '. 

available under aCC-BY-ND 4.0 International license.

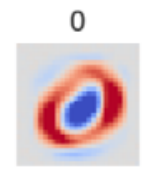

3

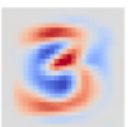

6

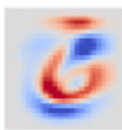

9

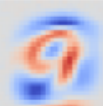

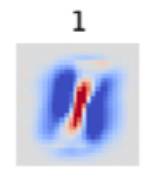

4

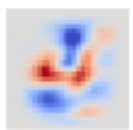

7

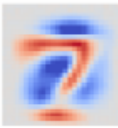

-

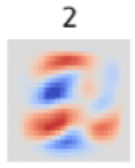

5

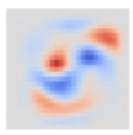

8

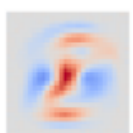

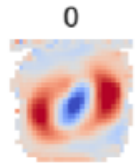

3
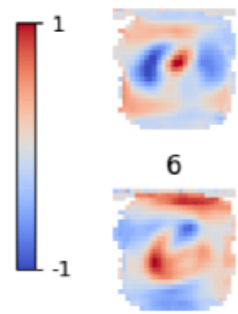

9

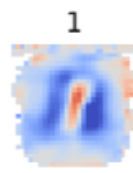

4

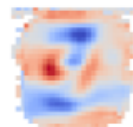

7

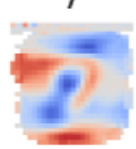

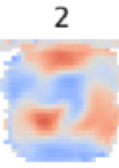

5

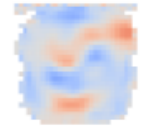

8

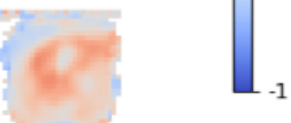

Fig. 4. Canonical features maps for a CNN classifying handwritten digits. The maps show the d'

322

323

324

325

326

sensitivity index for each point of the input space, obtained with Method 2a) (left) and Method 2b) (right). The red portions of the maps indicate the input features most associated with a given class. They visually resemble each digit, more or less blurred. The blue portions of the maps indicate the input features that are most reliably not present for a given class.

\subsection{Speech vs. music}

In this second example, we classified audio samples in a speech versus music task. We used the GTZAN database composed of 132 excerpts of speech and music (Tzanetakis \& Cook, 2002). The database was preprocessed to create samples with a fixed duration of 5 seconds, leading to a dataset of 768 samples. Those samples were randomly separated into a training set (691 excerpts) and a test set (77 excerpts, $10 \%$ of the dataset).

Following Patil et al. (2012), who performed an automatic classification of the musical timbre of short audio samples, sounds were first processed by an auditory model (Chi et al., 2005). The idea is to cast the input space into a representation that is interpretable in terms of auditory processing, unlike the raw waveform representation. Briefly, a filterbank corresponding to cochlear tonotopy is initially applied, followed by a 2-D Fourier analysis of the resulting time-frequency representation.

The model nutnut thus renresents temnoral modulations and snectral modulations contained in the 
bioRxiv preprint doi: https://doi.org/10.1101/2020.06.22.165688; this version posted June 23, 2020. The copyright holder for this preprint

(which was not certified by peer review) is the author/funder, who has granted bioRxiv a license to display the preprint in perpetuity. It is made available under aCC-BY-ND 4.0 International license.

input sound (Chi et al., 2005, Elliot \& Theunissen, 2009). The 4-D resulting arrays, with dimensions

of time, frequency, scale of spectral modulations, and rate of temporal modulation, are termed here

Spectro-Temporal Modulation representations (STM). We averaged the time dimension over the 5s

of each sample. Next, we applied a PCA to reduce dimensionality (30976 dimensions in our

implementation: 128 frequency channels x 11 scales x 22 rates, reduced to150 dimensions to preserve

$98 \%$ of the variance).

For classification, the output of the reduced PCA was fed to a Support Vector Machine (SVM)

with a Radial Basis Function (RBF). All of these steps are identical to Patil et al. (2012), to which the

reader is referred to for further details, as the specifics of the classifier are not critical to illustrate the

probing method. Briefly, a grid search on the RBF was performed to determine the best set of

parameters and the classifier accuracy was tested with a 10-fold cross-validation. We obtained an

average classification accuracy, i.e. whether the classifier is classifying the STM of a sound to the

correct music or speech class, of $94 \%$ (SD $=6 \%$ ) with the 10 -fold cross-validation and $98 \%$ on the

test set.

Figure 5 shows the discriminative feature maps for the speech versus music classification task.

For each case, we used 691 probing samples and 30 bubbles with standard deviation of $10 \mathrm{~Hz}$ in the

frequency dimension, $6 \mathrm{~Hz}$ in the rate dimension, and 3 cycles/octave in the scale dimension. As the

task is a binary classification, the maps for speech and music are simply mirror images of each other.

The discriminative regions of the auditory model STM representation appear to be mostly visible in

range around $500 \mathrm{~Hz}$, corresponding roughly to the position of the first formant in speech (Peterson

\& Barney, 1952). For the other dimensions, the classification depends on slow positive rates and low 
bioRxiv preprint doi: https://doi.org/10.1101/2020.06.22.165688; this version posted June 23, 2020. The copyright holder for this preprint (which was not certified by peer review) is the author/funder, who has granted bioRxiv a license to display the preprint in perpetuity. It is made available under aCC-BY-ND 4.0 International license.

In the case of the Method $1 \mathrm{~b}$ ), with the training set unavailable, a first probing was attempted

with a uniform white noise but failed to provide classification decisions sampling the two categories:

all noises were classified as music, a perhaps amusing finding which we will not develop here. The

uniform white noise was thus replaced by a pseudo random noise generated in a PCA-reduced
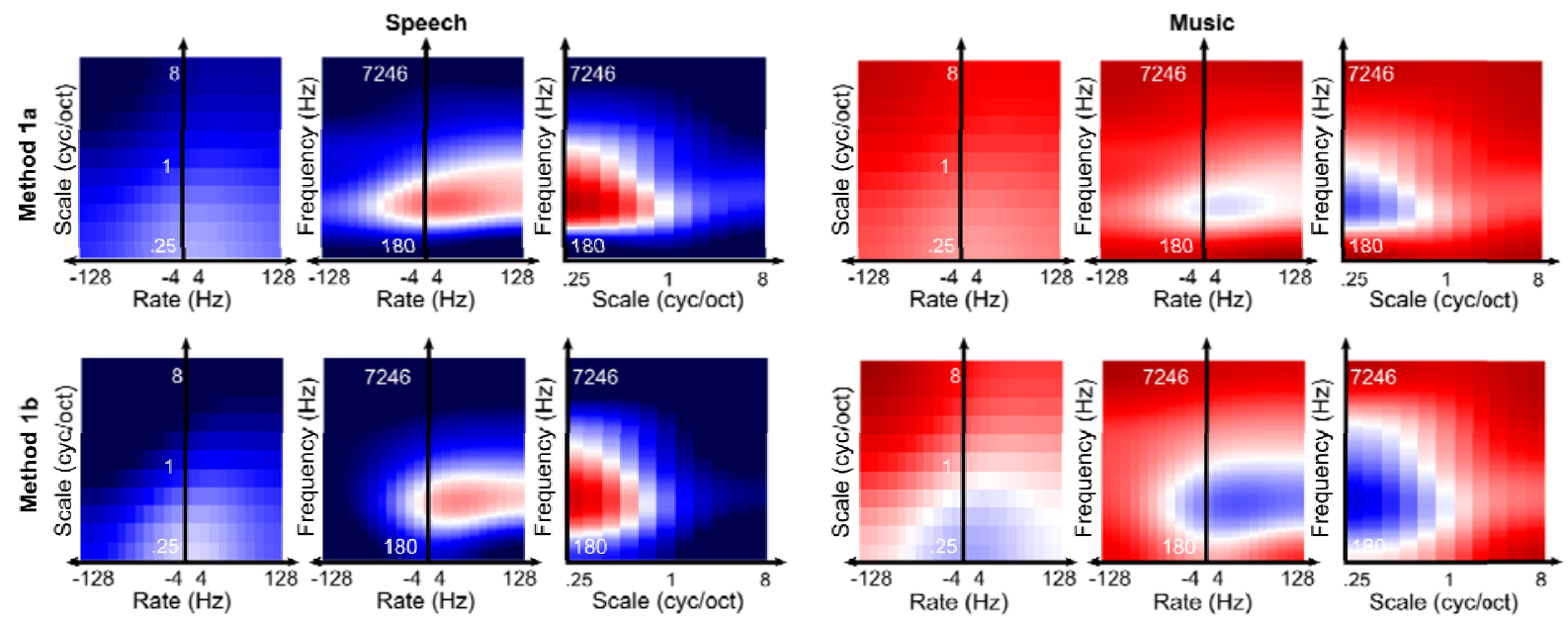

Fig. 5. Discriminative maps for speech and music in the STM representation. The 4-D STM

representations are projected in the three dimensions (frequency, scale, rates), and expressed in $d B$.

Method 1a) (left) and method 1b) (right). The complete STM matrices are available in supplementary

Figure S1. Method 1a) uses the training set while Method 1b) uses pseudo random noise. The red

class. The blue regions correspond to less important features. As this is a binary classification task,

the speech and music masks are simply opposite versions of each other.

Figure 6 shows the canonical feature maps for the speech versus music classification task. Compared 
bioRxiv preprint doi: https://doi.org/10.1101/2020.06.22.165688; this version posted June 23, 2020. The copyright holder for this preprint (which was not certified by peer review) is the author/funder, who has granted bioRxiv a license to display the preprint in perpetuity. It is made available under aCC-BY-ND 4.0 International license. the STM representation, or, similarly, they indicate the "average" speech and music sounds learnt by the SVM. For speech, some formantic structure is visible on the frequency dimension, associated with low rates typical of prosodic modulations (middle panels). These formantic regions extend to higher scales (right panels), perhaps because formants are superimposed on a harmonic structure during vowel sounds. Conversely, musical sounds more typically contain high modulation rates and spectral scales. These observations are consistent with previous analyses of STM representations (Elliott \& Theunissen, 2009; Chi et al., 2005). These observations are consistent with previous analyses of STM representations (Elliott \& Theunissen, 2009; Chi et al., 2005). Again, the canonical features observed for speech and music are complementary by construction with our method. It should be noted that, as intuitively expected, canonical features depend on the acoustic characteristics of speech and music, but they also depend on the task of the classifier. Probing a classifier trained to discriminate speech from e.g. environmental sounds would likely provide different canonical features for speech. This result may seem like a limitation of the method, but it also highlights the way an automatic classifier performs a binary task. This may be an important difference to keep in mind when comparing classifiers with human perception, which has to perform many concurrent tasks in parallel. Yet, "opportunistic features" that depend both on sensory information and the task at hand have been suggested for auditory timbre recognition, a task not unlike the one probed here (Agus et al., 2019), a task not unlike the one probed here.
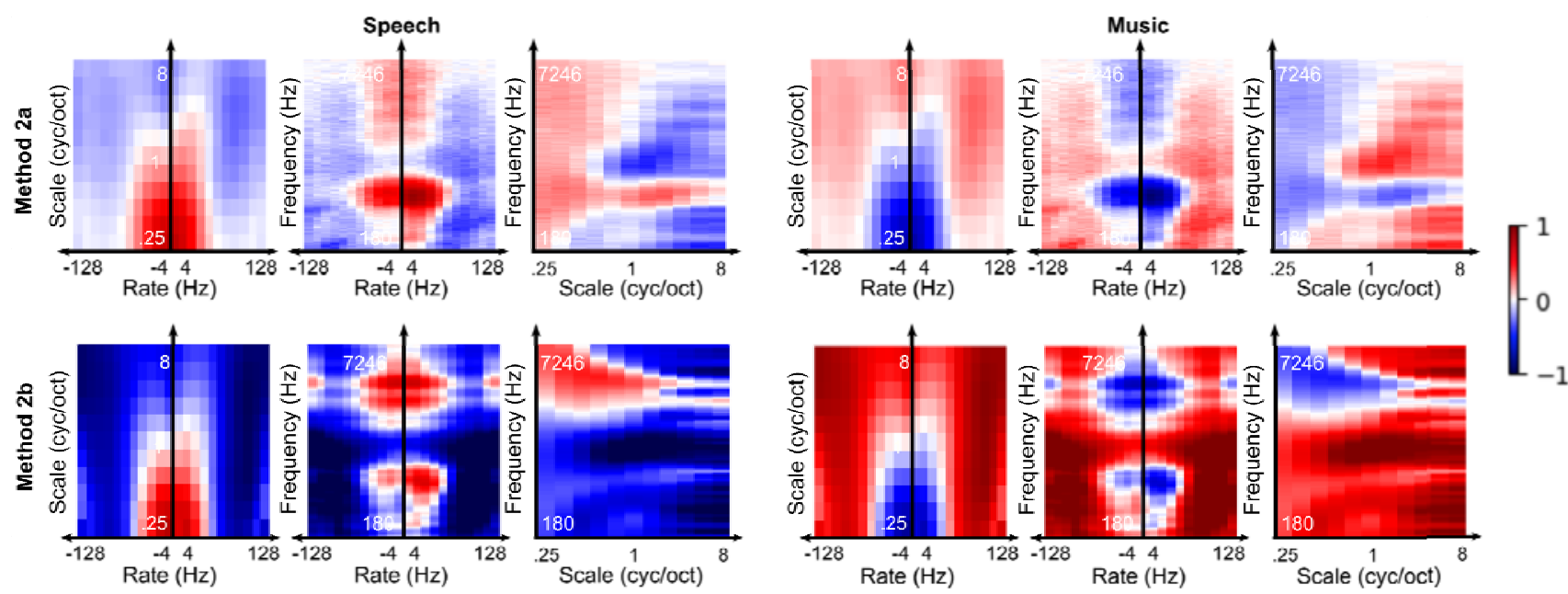

available under aCC-BY-ND 4.0 International license.

409 Fig. 6. Canonical STM representations for speech and music. The d' sensitivity index is displayed

410 for projections of the 4-D representation. The complete STM matrices are illustrated in

411 supplementary Figure S2. The red regions of the maps indicate features most often encountered

412 within each category, whereas the blue regions indicate features most often not encountered within

413 each category.

414 3. Discussion

$415 \quad 4.1$ Summary

416 The method presented in this paper used a reverse correlation framework to visualize the input

417 features discovered by an automatic classifier to reach its decisions. When the classifier is successful, such features should provide insights about the structure of the input dataset. Over two examples using different kinds of classifiers (a CNN and an SVM with RBF) and using different kinds of input representations (2-D visual images and 1-D audio samples converted to a 4-D auditory model), we illustrated how the method could highlight relevant aspects of a classifier's operation. Moreover, by combining standard noise perturbation techniques with so-called bubbles (Gosselin \& Shyns, 2002), we showed that the probing method can be focused either on discriminative features, related to the decision strategy of the classifier, or on canonical features, related to the output classes' main characteristics.

\subsection{Benefits}

In the context of neuroscience and experimental psychology, there are benefits in using a reverse correlation framework to interpret classifiers, as a way to complement other more specialized machine-learning interpretation techniques (Zhou et al., 2016; Ribeiro et al., 2016; Petsiuk et al., 2018; Borji \& Lin, 2019; Xu et al., 2018). experimental psychology. It has proved useful to gain insights about stimulus features relevant to 
436 classifiers amounts to translating and applying a familiar toolbox to another, conceptually similar

437 problem of characterizing a black-box system.

438 Second, the method is by design fully agnostic by design. It operates on the input space of the 439 classifier, whatever this space might be. It does not make assumptions on the classifier's architecture 440 or inner operations. Focusing on the input space rather than the classifier's architecture is especially

441 desirable in situations where the classifier is not the main interest of study, but rather, the structure of 442 the input dataset is.

Third, it can be applied to classifiers that have not been designed by the user, as it does not even require the availability of the training dataset. Access to labeled input data is helpful in improving the efficiency of the method, for instance by allowing to shape the perturbation noise, but this is a mild constraint: there are no interesting situations we can think of for which both the classifier and the type of data to classify would be unknown. the input space. Such a representation should make intuitive sense to the user of the method, and the features discovered can be interpreted a posteriori in terms of attributes of the stimuli. If the representation does not make intuitive sense, then one possible benefit of the method is to help recast the input space into a more meaningful representation, as was done here in the audio example for which the waveform samples were pre-processed with an auditory model. This idea is further detailed in the "Perspectives" subsection.

\subsection{Limitations}

There are also limitations associated to the use of a reverse correlation approach to interpret automatic classifiers. Broadly speaking, these limitations follow those already described for reverse correlation in neuroscience.

First, reverse correlation is inspired from the analysis of linear systems, whereas machine- 
bioRxiv preprint doi: https://doi.org/10.1101/2020.06.22.165688; this version posted June 23, 2020. The copyright holder for this preprint

(which was not certified by peer review) is the author/funder, who has granted bioRxiv a license to display the preprint in perpetuity. It is made available under aCC-BY-ND 4.0 International license.

464 \& Heeger, 2002). Such extensions could be applied to the interpretation of classifier's features.

465 Interestingly, the reverse correlation approach bears some similarities with the "distillation" method

466 from the machine learning literature (Hinton et al., 2015). Distillation consists in mimicking the

467 behavior of a black-box classifier with an easily-interpretable classifier, such as a linear one (linear

468 SVM, etc.). Both techniques can thus be viewed as attempting to find linear approximations of a

469 classifier's operations, but their precise relationship remain to be investigated.

470 Second, the method has a number of parameters the number and size of bubbles, the space to

471 generate the probing noise with reduction dimension methods such as PCA when the training set is

472 available, which are not algorithmically constrained. In the examples above, the parameter space was

473 explored heuristically. One suggested heuristic was to try and cover the output classes in a balanced

474 manner with the probing set. However, even though statistical tests of the resulting features are

475 available, we do not provide any fitness criterion, i.e. a way to quantify the efficiency of the method

476 for a given set of parameters, for the features obtained with the method. Rather, we would argue that

477 the iterative process for parameter tuning can be part of the interpretation process since finding the

478 right probing structure provides some information on the structure of the dataset. Also, assessing

479 whether the discovered features make intuitive sense relies mostly on the knowledge and goals of the user. Thus, it may not be easily formalized into a fitness criterion. If more formally defined methods are needed, either from the outset or after a first exploration of the classifier with reverse correlation, other classifier-specific tools exist (e.g., Zhou et al., 2016; Ribeiro et al., 2016; Petsiuk et al., 2018).

Third, the method implicitly assumes that there are no invariances by translation or otherwise in the classifier's algorithm. With reverse correlation, each point of the input space is treated independently of all others, so a feature discovered in one sub-part of the input space will not impact other, perhaps similar features in other sub-parts. This assumption is obviously falsified by CNN architectures, which are purposely designed to incorporate such invariances. In the CNN example illustrated here with digits recognition, this limitation was circumvented by the fact that all digits in the probing set were roughly spatially aligned. For the SVM on audio data, a time-averaging over the time dimension achieved a similar effect. Thus, a mitigation strategy is available: a rough alignment 
492 produce meaningful results. Another possible direction to address these invariance issues is to

493 generate the probing noise in an appropriate space. Using a PCA partly achieves this. Finally, using

494 another representation space with built-in invariances, e.g. by using wavelets transforms, can be

495 considered.

\section{$496 \quad 4.3$ Perspectives}

497 The probing method is technically applicable to any classifier's architecture with any kind of input data. It is thus beyond the scope of this final section to list all possible use cases in the context of neuroscience. We will simply provide a few suggestions, to illustrate the kind of problems that could benefit from the probing method. is the exploration of the input representation fed to the classifier. The hypothesis is that, the more appropriate the representation, the more explainable the classifier should be. For instance, one could assume that the massively non-linear transformations of auditory and visual information that characterize perceptual systems serve to build a stimulus manifold within which perceptual boundaries are approximately linear (Georgopoulos et al., 1986; Jazayeri \& Movshon, 2006; Kell et al., 2018). So, with the correct representation, a classifier modeling a perceptual decision process should be easily interpretable, or at least more easily interpretable than if the input representation was not reflecting perceptual processing. It is with this hypothesis in mind that the audio samples of the example illustrated above were first processed with an auditory model. Even though there are successful deep learning models operating on the raw audio waveforms (e.g. Wavenet, Oord et al., 2006), it is not expected that interpreting them in terms of waveform features will be meaningful. For instance, inaudible phase shifts between frequency components in the input would impact the waveform representation, but should not change the classifier's decision. An auditory model, in contrast, incorporates transforms inspired by the neurophysiology of the hearing system. If the features extracted resemble those available to a human observer, then they should be revealed when

517 probing a classifier. In fact, the ease of interpreting a classifier feature could be a proxy to evaluate an input representation's adequation to a perceptual task. 
bioRxiv preprint doi: https://doi.org/10.1101/2020.06.22.165688; this version posted June 23, 2020. The copyright holder for this preprint

(which was not certified by peer review) is the author/funder, who has granted bioRxiv a license to display the preprint in perpetuity. It is made available under aCC-BY-ND 4.0 International license.

Another possible application is when building “ideal observer" models (Geisler, 2004). The

idea of an ideal observer model is to compute the best theoretical performance on a task, given a set

521 of assumptions (classically, endowing the ideal observer with unbiased decision criteria, perfect and

unlimited memory, and so on). This upper performance boundary is then compared to the observed

performance with human participants or neural recordings. When considering classification or

discrimination tasks, and when a formal model of the ideal observer is unavailable, it can be of

interest to build pseudo-ideal observer models with machine learning classifiers. The advantage of

our probing method is then that the classifier's strategy can be directly compared to a reverse

correlation analysis of neural or psychophysical data, to ask whether the classifier and the

experimental observer used the same decision features.

Finally, the general benefits of interpreting classifiers also apply to the field of neuroscience.

In a broad sense, probing is intended to help an expert making sense of a classifier's strategy. If the

features discovered through probing fit a theoretical model, this would reassure the expert that the

performance relies on reasonable principles, which is especially important in clinical applications. In

return, the expert's intuition may also help improve the classifier, for instance by simplifying its input

representation through pre-processing, and so hopefully making it less brittle to irrelevant variations

in input that may have been picked up by overfitting during training (Goodfellow et al., 2015). The

discriminative features could be particularly useful to reduce the complexity of a classifier. Based on

the discriminative features map, it may be possible to select a subset of important and intelligible

features, which can then be used to build a more computationally efficient classifier, for very large dataset and/or for real-time processing.

\section{Conclusions}

541 We presented a novel method to interpret machine-learning classifiers, with the aim that the method should be agnostic and well-suited to applications in the neuroscience domain. Based on the reverse correlation framework, the method uses stochastic perturbation of inputs to observe the classifier's 
bioRxiv preprint doi: https://doi.org/10.1101/2020.06.22.165688; this version posted June 23, 2020. The copyright holder for this preprint (which was not certified by peer review) is the author/funder, who has granted bioRxiv a license to display the preprint in perpetuity. It is made available under aCC-BY-ND 4.0 International license.

547 benefits and limitations as reverse correlation when applied to psychophysical or neural data. Our

548 hope is that such a method can provide a simple and generic interface between neuroscientists and

549 machine-learning tools.

550 
551

552

553

554

555

556

557

558

559

560

561

562

563

564

565

566

567

568

569

570

571

572

573

574

575

576

577

578

\section{References}

Ahumada Jr, A., \& Lovell, J. (1971). Stimulus features in signal detection. The Journal of the Acoustical Society of America, 49(6B), 1751-1756. https://doi.org/10.1121/1.1912577

Adolphs, R., Gosselin, F., Buchanan, T. W., Tranel, D., Schyns, P., \& Damasio, A. R. (2005). A mechanism for impaired fear recognition after amygdala damage. Nature, 433(7021), 68-72. https://doi.org/10.1038/nature03086

Arnal, L. H., Flinker, A., Kleinschmidt, A., Giraud, A. L., \& Poeppel, D. (2015). Human screams occupy a privileged niche in the communication soundscape. Current Biology, 25(15), 20512056.https://doi.org/10.1016/j.cub.2015.06.043

Benjamini, Y., \& Hochberg, Y. (1995). Controlling the false discovery rate: a practical and powerful approach to multiple testing. Journal of the Royal statistical society: series B (Methodological), 57(1), 289-300. https://doi.org/10.1111/j.2517-6161.1995.tb02031.x

Borji, A., \& Lin, S. (2019). White Noise Analysis of Neural Networks. arXiv preprint arXiv:1912.12106.

Chi, T., Ru, P., \& Shamma, S. A. (2005). Multiresolution spectrotemporal analysis of complex sounds. The Journal of the Acoustical Society of America, 118(2), 887-906. https://doi.org/10.1121/1.1945807

Elhilali, M. (2019). Modulation Representations for Speech and Music. In Timbre: Acoustics, Perception, and Cognition (pp. 335-359). Springer. https://doi.org/10.1007/978-3-030-14832$4 \_12$

Elliott, T. M., \& Theunissen, F. E. (2009). The modulation transfer function for speech intelligibility. PLoS computational biology, 5(3). https://doi.org/10.1371/journal.pcbi.1000302

Eggermont, J. J., Johannesma, P. I. M., \& Aertsen, A. M. H. J. (1983). Reverse-correlation methods in auditory research. Quarterly reviews of biophysics, 16(3), 341-414. https://doi.org/10.1017/s0033583500005126

Deng, L. (2012). The mnist database of handwritten digit images for machine learning research [best of the web]. IEEE Signal Processing Magazine, 29(6), 141- 
579 Geisler, W. S. (2004). "Ideal Observer analysis," in Visual Neurosciences, eds L. Chalupa and J.

$580 \quad$ Werner (Boston, MA: MIT press), 825-837.

581 Georgopoulos, A. P., Schwartz, A. B., \& Kettner, R. E. (1986). Neuronal population coding of movement direction. Science, 233(4771), 1416-1419. https://doi.org/10.1126/science.3749885

Gosselin, F., \& Schyns, P. G. (2002). RAP: A new framework for visual categorization. Trends in

Gosselin, F., \& Schyns, P. G. (2001). Bubbles: a technique to reveal the use of information in recognition tasks. Vision research, 41(17), 2261-2271. https://doi.org/10.1016/s00426989(01)00097-9

Jordan, M. I., \& Mitchell, T. M. (2015). Machine learning: Trends, perspectives, and prospects. Science, 349(6245), 255-260. https://doi.org/10.1126/science.aaa8415

Gosselin, F., \& Schyns, P. G. (2003). Superstitious perceptions reveal properties of internal representations. Psychological science, 14(5), 505-509. https://doi.org/10.1111/14679280.03452

Goodfellow, I. J., Shlens, J., \& Szegedy, C. (2014). Explaining and harnessing adversarial examples. arXiv preprint arXiv:1412.6572. https://doi.org/10.5220/0006123702260234

Green, D. M., \& Swets, J. A. (1966). Signal detection theory and psychophysics (Vol. 1). New York: Wiley.

Guidotti, R., Monreale, A., Ruggieri, S., Turini, F., Giannotti, F., \& Pedreschi, D. (2018). A survey of methods for explaining black box models. ACM computing surveys (CSUR), 51(5), 1-42. https://doi.org/10.1145/3236009

Hinton, G., Vinyals, O., \& Dean, J. (2015). Distilling the knowledge in a neural network. arXiv preprint arXiv:1503.02531.

Jazayeri, M., \& Movshon, J. A. (2006). Optimal representation of sensory information by neural populations. Nature neuroscience, 9(5), 690-696. https://doi.org/10.1038/nn1691

Kell, A. J., Yamins, D. L., Shook, E. N., Norman-Haignere, S. V., \& McDermott, J. H. (2018). A task-optimized neural network replicates human auditory behavior, predicts brain responses, 

https://doi.org/10.1016/j.neuron.2018.03.044

King, J. R., \& Dehaene, S. (2014). Characterizing the dynamics of mental representations: the temporal generalization method. Trends in cognitive sciences, 18(4), 203-210. https://doi.org/10.1016/j.tics.2014.01.002

Kriegeskorte, N., \& Douglas, P. K. (2018). Cognitive computational neuroscience. Nature neuroscience, 21(9), 1148-1160. https://doi.org/10.1038/s41593-018-0210-5

LeCun, Y., Bengio, Y., \& Hinton, G. (2015). Deep learning. nature, 521(7553), 436-444. https://doi.org/10.1038/nature14539

Lillicrap, T. P., \& Kording, K. P. (2019). What does it mean to understand a neural network?. arXiv preprint arXiv:1907.06374.

Maris, E., \& Oostenveld, R. (2007). Nonparametric statistical testing of EEG-and MEG-data. Journal of neuroscience methods, 164(1), 177-190. https://doi.org/10.1016/j.jneumeth.2007.03.024

Neri, P., Parker, A. J., \& Blakemore, C. (1999). Probing the human stereoscopic system with reverse correlation. Nature, 401(6754), 695-698. https://doi.org/10.1038/44409

Neri, P., \& Heeger, D. J. (2002). Spatiotemporal mechanisms for detecting and identifying image features in human vision. Nature neuroscience, 5(8), 812-816. https://doi.org/10.1038/nn886

Neri, P., \& Levi, D. M. (2006). Receptive versus perceptive fields from the reverse-correlation viewpoint. Vision research, 46(16), 2465-2474. https://doi.org/10.1016/j.visres.2006.02.002

Oord, A. V. D., Dieleman, S., Zen, H., Simonyan, K., Vinyals, O., Graves, A., ... \& Kavukcuoglu, K. (2016). Wavenet: A generative model for raw audio. arXiv preprint arXiv:1609.03499.

Paquette, S., Takerkart, S., Saget, S., Peretz, I., \& Belin, P. (2018). Cross-classification of musical and vocal emotions in the auditory cortex. Ann. NY Acad. Sci, 1423, 329-337. https://doi.org/10.1111/nyas.13666

Patil, K., Pressnitzer, D., Shamma, S., \& Elhilali, M. (2012). Music in our ears: the biological bases of musical timbre perception. PLoS computational biology, 8(11). https://doi.org/10.1371/journal.pcbi.1002759 
633 Peterson, G. E., \& Barney, H. L. (1952). Control methods used in a study of the vowels. The Journal

634 of the acoustical society of America, 24(2), 175-184. https://doi.org/10.1121/1.1906875

635 Petsiuk, V., Das, A., \& Saenko, K. (2018). Rise: Randomized input sampling for explanation of

636 black-box models. arXiv preprint arXiv:1806.07421.

637 Richards, B. A., Lillicrap, T. P., Beaudoin, P., Bengio, Y., Bogacz, R., Christensen, A., ... \& Gillon,

638 C. J. (2019). A deep learning framework for neuroscience. Nature neuroscience, 22(11), 17611770. https://doi.org/10.1038/s41593-019-0520-2

Ribeiro, M. T., Singh, S., \& Guestrin, C. (2016). " Why should i trust you?" Explaining the predictions of any classifier. In Proceedings of the 22nd ACM SIGKDD international conference on knowledge discovery and data mining (pp. 1135-1144). https://doi.org/10.1145/2939672.2939778

Ringach, D., and Shapley, R. (2004). Reverse correlation in neurophysiology. Cogn. Sci. 28, 147-

Tzanetakis, G., \& Cook, P. (2002). Musical genre classification of audio signals. IEEE Transactions on speech and audio processing, 10(5), 293-302. https://doi.org/10.1109/tsa.2002.800560

Venezia, J. H., Hickok, G., \& Richards, V. M. (2016). Auditory “bubbles": Efficient classification of the spectrotemporal modulations essential for speech intelligibility. The Journal of the Acoustical Society of America, 140(2), 1072-1088. https://doi.org/10.1121/1.4960544 metastatic breast cancer. arXiv preprint arXiv:1606.05718. 
661 Xu, T., Garrod, O., Scholte, S. H., Ince, R., \& Schyns, P. G. (2018). Using psychophysical methods

662 to understand mechanisms of face identification in a deep neural network. In Proceedings of the

663 IEEE Conference on Computer Vision and Pattern Recognition Workshops (pp. 1976-1984).

664 https://doi.org/10.1109/cvprw.2018.00266

665 Zihni, E., Madai, V. I., Livne, M., Galinovic, I., Khalil, A. A., Fiebach, J. B., \& Frey, D. (2020).

666 Opening the black box of artificial intelligence for clinical decision support: A study predicting

667 stroke outcome. Plos one, 15(4), e0231166.https://doi.org/10.1371/journal.pone.0231166

668 Zhou, B., Khosla, A., Lapedriza, A., Oliva, A., \& Torralba, A. (2016). Learning deep features for

669 discriminative localization. In Proceedings of the IEEE conference on computer vision and

670 pattern recognition (pp. 2921-2929). https://doi.org/10.1109/cvpr.2016.319

671

672

673 Acknowledgements: Research supported by grants ANR-16-CONV-0002 (ILCB), ANR-11-LABX-

6740036 (BLRI) and the Excellence Initiative of Aix-Marseille University (A*MIDEX) (ET), ANR-10-

675 LABX-0087 IEC and ANR-10-IDEX-0001-02 PSL (DP). TA was supported by the Human Frontier

676 Science Program (LT000362/2018-L).

677 
bioRxiv preprint doi: https://doi.org/10.1101/2020.06.22.165688; this version posted June 23, 2020. The copyright holder for this preprint (which was not certified by peer review) is the author/funder, who has granted bioRxiv a license to display the preprint in perpetuity. It is made available under aCC-BY-ND 4.0 International license.

\section{8}

679

680

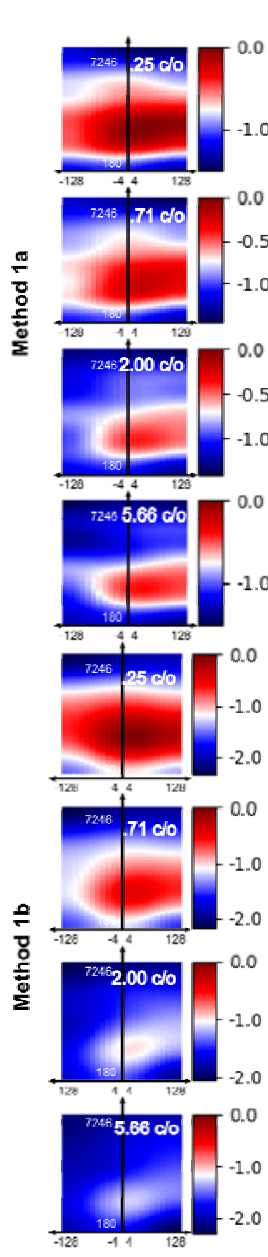

\section{Supplementary Materials}

Figure S1. Complete Spectro-Temporal Modulation representations discriminative maps, in dB, for speech vs. music classification task.
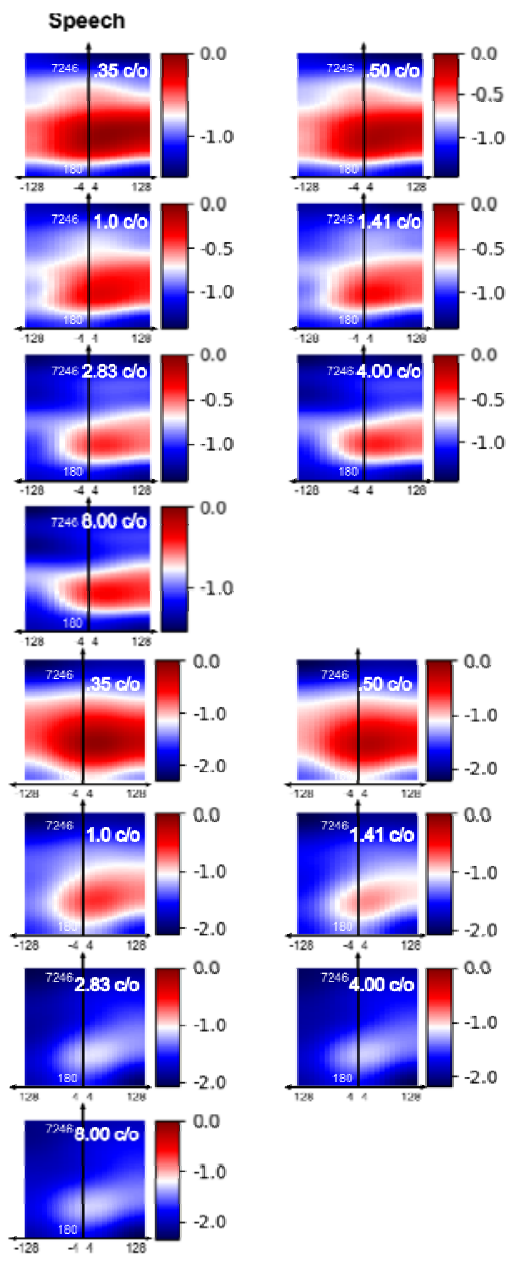
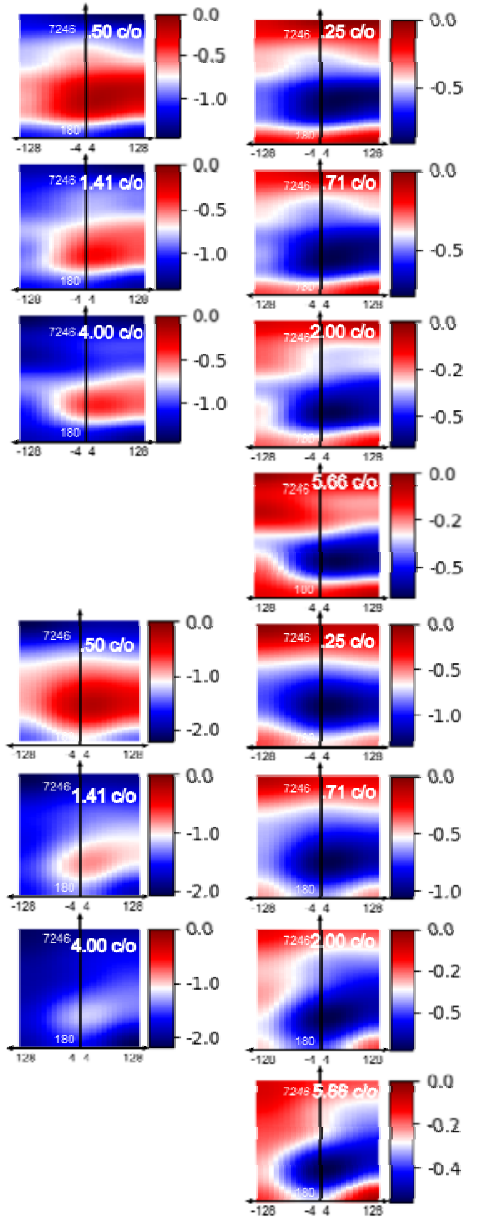
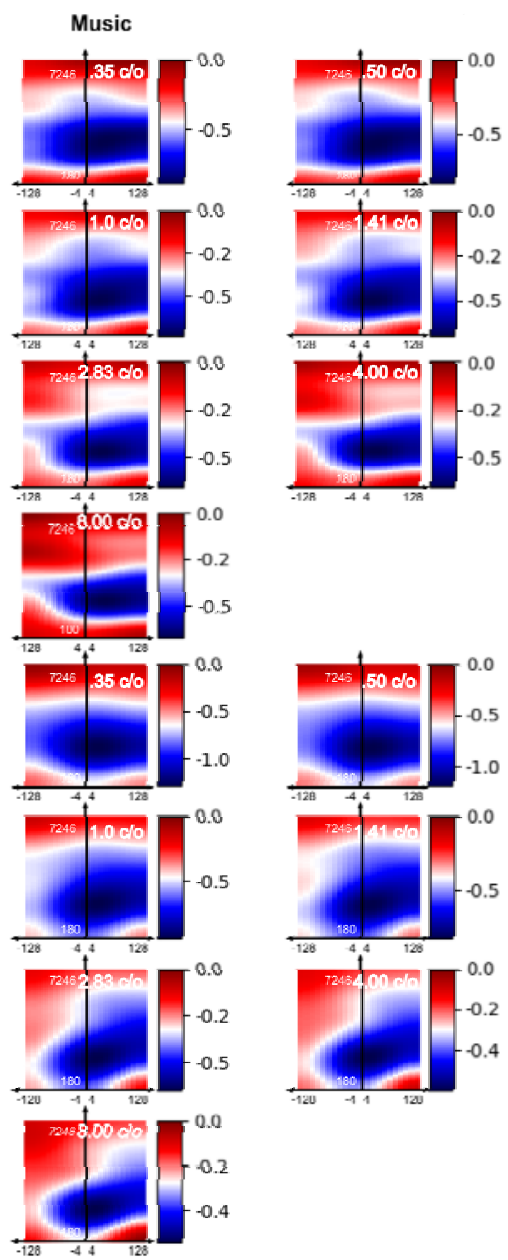

682 
bioRxiv preprint doi: https://doi.org/10.1101/2020.06.22.165688; this version posted June 23, 2020. The copyright holder for this preprint (which was not certified by peer review) is the author/funder, who has granted bioRxiv a license to display the preprint in perpetuity. It is made available under aCC-BY-ND 4.0 International license.

690 Figure S2. Complete Spectro-Temporal Modulation representations canonical maps (d'), for speech

691 vs. music classification task.
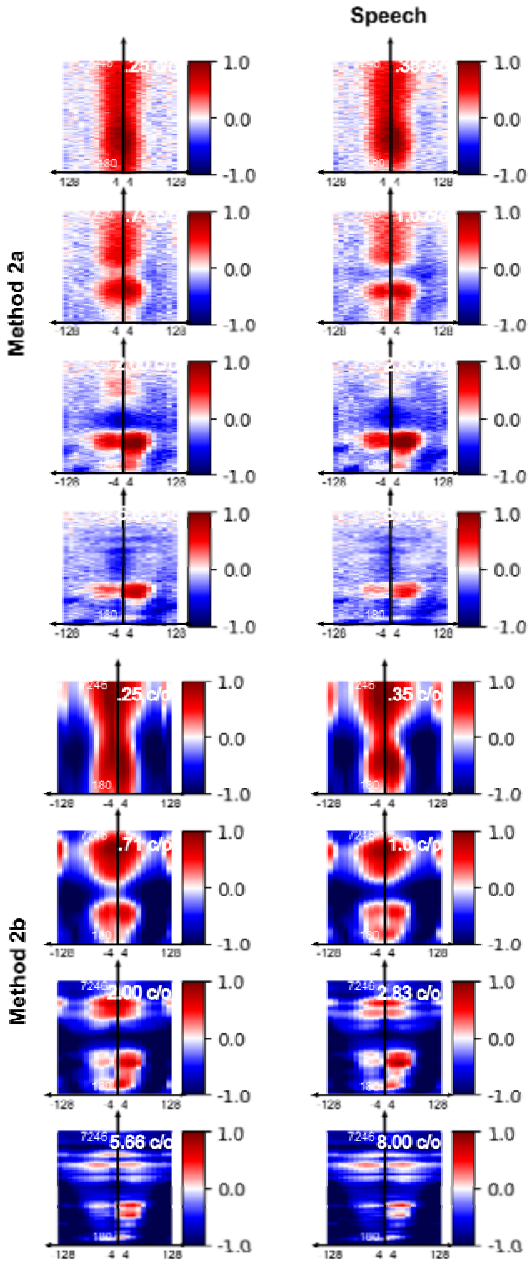
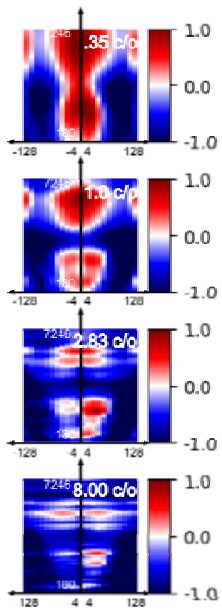
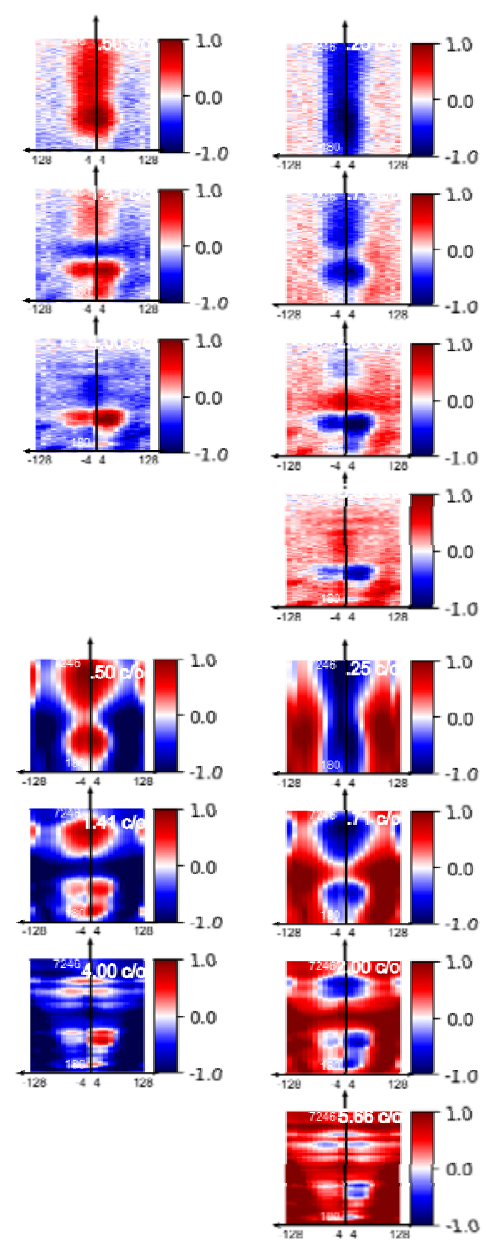
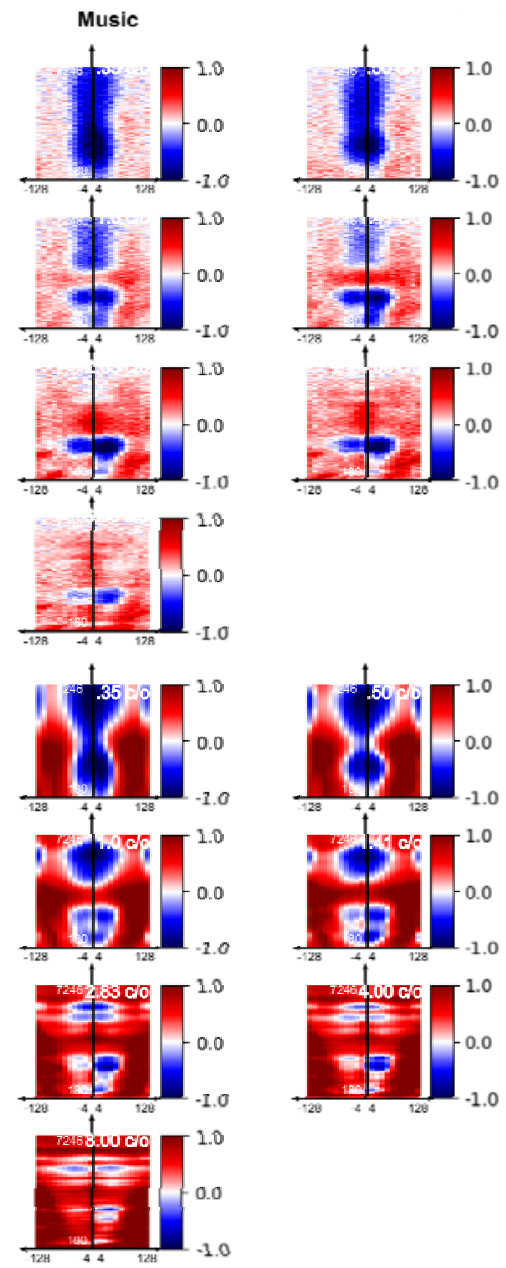